\title{
Non-medicinal helio-magnetoprotective remedies against the influence of solar and geomagnetic disturbances on human physiological parameters
}

\author{
Alexander Trofimov \\ International Scientific-Research Institute of Cosmic Anthropoecology, Prospect Academician Lavrentiev, Novosibirsk, Russia \\ Email address: \\ isrica2@rambler.ru
}

\section{To cite this article:}

Alexander Trofimov. Non-Medicinal Helio-Magnetoprotective Remedies Against the Influence of Solar and Geomagnetic Disturbances on Human Physiological Parameters. Clinical Medicine Research. Vol. 2, No. 4, 2013, pp. 63-67. doi: 10.11648/j.cmr.20130204.15

\begin{abstract}
The biotropic influence of ionospheric electrical currents (electrojets) and electromagnetic disturbances in the zone of the auroral oval on the electrical and cardiovascular homeostasis of healthy people was the topic of research. This was an investigation of non-medicinal helio-magnetoprotective remedies. "Helio-magnetoprotective" drinking water, certified and patented in Russia, has been developed for and tested in the circumpolar region (town Dixon) on inhabitants-volunteers with high level of helio-magnetosensitivity. This water after exposure to the repeatedly weakened magnetic field of the Earth ( device TRODR) substantially changes its structure, forming, on data of high effective chromatography, resistant nanocluster formations with marked biocatalytic properties which are enhanced with the use of the special informational holograms. Drinking water is treated using translucent holograms in this device is innovation technology. Helio-magnetoprotective water, according to our data, appears to be able to vary the degree of functional dependence of cardiovascular and other systems of both healthy and sick people on extreme impacts of heliogeophysical factors. Protective effect was assessed by changing in the vector of significant correlations: direct dependencies after drinking water became significantly reversed. Electrocardiographic parameters (R, PQ, QT), electrical conductivity in the cutaneous projections of acupuncture points, heart rate, and the blood pressure of the test subjects were registered three times: in the background conditions, 10 minutes after taking control water, and 10 minutes after receiving the same amount of helio-magnetoprotective water. The intensity of the polar electrojets and cosmic noise was determined by the methods of vertical sounding of the ionosphere and the riometer. The state of the polar magnetosphere was estimated according to the value of the Ak-index. It has been shown that the electrical and hemodynamic parameters of healthy people in the Far North are significantly altered during the periods of increased magnetic-ionospheric activity. Based on additional magnetic testing, the helio-magnetosensitivity of an organism increases during periods of increased level of cosmic noises as registered by riometers. Use of helio-magnetoprotective drinking water reduces the biotropic impacts of cosmophysical factors on human functional systems. The use of helio-magnetoprotective drinking water for prophylactic measures is effective against hello-dependent diseases, crisis states, and their life-threatening hemodynamic consequences for people living near the world's Polar Regions and an other places.
\end{abstract}

Keywords: Polar Electrojets,Magnetic Disturbances,Helio-Magnetosensitivity, Helio-Magnetoprotective Drinking Water

\section{Background}

At present, the assumption of geophysicists about jerks and secular excursions in the Earth's magnetic field, accompanied by a decrease in its induction an average of $40-70 \mathrm{nT}$ per year is confirmed $[1,2,3]$. Since the beginning of the 21 st century, our planet has entered just this phase-gradual weakening of geomagnetic induction and consequent reduction of the buffering properties of the magnetosphere. Each year more and more charged solar and galactic particles, which had usually been reflected by the magnetosphere, rush to Earth. A new situation that is very dangerous to the cosmophysical biosphere is created, in which the protons, electrons, and particles secondarily ionized in the atmosphere reach the living zones on Earth and most of the areas occupied by humans.

The medical consequences of increasing solar and geomagnetic disturbances are widely discussed in the scientific and medical community $[4,5,6]$.

Under these conditions, advanced scientific investigations 
using the method of experimental geomagnetic deprivation appeared to be extremely apropos. Just these studies were begun in 1999 in the Siberian Branch of the Russian Academy of Medical Sciences (Novosibirsk) under the leadership of its founder, Academician Vlail Kaznacheev, one of the pioneers of a new cycle of scientific research in the Far North [7], and then continued by his disciples [8].

The structural features of the Earth's magnetic field in the Polar Regions, remaining "uncovered" by the magnetic force lines, allow the regions of the Far North to be considered a natural experimental platform because solar-cosmic energy flows reach areas inhabited by people almost unimpeded.

We considered the development of effective protection for the health of inhabitants of the Far North to be a priority task At the 14th International Congress on Circumpolar Health (ICCH-14) in Canada, our organization-International Scientific Research Institute of Cosmic Anthropoecology (ISRICA) - presented materials tying the duration of human life in the Far North to the individual helio-magnetosensitivity as formed in prenatal ontogenesis and to the intensity of geophysical factors in the area of residence [9]. At ICCH-15, we proposed discussion of new data on possible non-medicinal protection of helio-magnetosensitive persons through the use of helio-magnetoprotective water produced by ISRICA technology in the TRODR installation.

Development of non-medicinal helio-magneto protection to decrease the direct impact of helio-physical factors on the human organism in the high and middle latitude regions was important as well.

The purpose of this study was to estimate impact of the helio-physical situation on human functional systems during life in high and middle latitude regions as well as to test the new non-medicinal helio-magnetoprotective drinking water on volunteers who are inhabitants of the circumpolar region at the end of the first year of their adaptation to Far North conditions.

\section{Material and Methods}

1. Original "helio-magnetoprotective" water was used, as developed on the basis of the following Russian inventions:

A. The means of possessing helio-magnetoprotective properties (patent RF № 2342149 of December 27, 2008).

B. The method for creating a hologram containing non-visualized physiologically significant information (patent RF № 2239860 of November 10, 2004) together with use of the portable hypogeomagnetic "TRODR-1" device (Authors: Alexander Trofimov, Gennady Drujinin, 2011).

Holograms are translucent films containing analog information about vitamins and antioxidants authorized for use in Russia. Information on their use in the preparation of drinking water was given to the volunteers who agreed to the testing. The portable hypogeomagnetic device TRODR-1 is a hollow chamber with a volume of 19 liters, whose walls are made of the composite material that shields the Earth's magnetic field. The installation is certified in Russia and permitted for the manufacture of water-based drinks. Drinking water is treated using translucent holograms in the device TRODR-1, which weakens the magnetic field of the Earth.

2. A person's helio-magnetosensitivity was determined using the method developed in the laboratory of helio-climatopathology of SCCEM SB RAMS (Aut. Cert. USSR 1396323 of January 15, 1988).

3. Electrocardiography was carried out on a certified portable belt thermal electrocardiograph with an estimate of R, PQ, QT parameters. Measurement of electrical conductivity (I, R) in the cutaneous projections of acupuncture points of the meridians of the heart and pericardium was performed on the apparatus PEP-1. A certified device PEP-1 was used for the assessment of the electrical conductivity dynamic (in microamperes) in cutaneous projections of acupuncture points (acupuncture). Heart rate and blood pressure of volunteers were measured on an electronic blood pressure tonometer.

4. The state of the magnetosphere was determined by the magnitude of Ak-index. Ak is an index that characterizes in relative units the degree of changes in the components of the Earth's magnetic field and its full vector.

5. Mathematical processing of the data was performed using the software package Statistica.6.0.

\section{Study Design}

We assessed physiological parameters of healthy men, aged $18-20$ years $(n=120$ in 1999 and $n=48$ in 2012). The subjects were assessed $8-10$ months after their arrival in the North from the middle-latitude regions of Russia. The subjects had different professions, mostly manual labor.

A comparative investigation of the volunteers took place during periods of quiet and disturbed magneto-ionosphere. Quiet and disturbed states of the magneto-ionosphere reflect a varying degree of fluctuations of geomagnetic induction and, in relation to the ionosphere, varying intensity of beams of charged particles.

Evaluation of a person's magnetosensitivity was carried out using a dosed magnetic load by a constant magnetic field (induction $20 \mathrm{mTl}$ ) in the domain of the ears with a wide representation of acupuncture points and nerve receptors. At all stages of the research, certified installations and medical devices were used.

As a result of the investigations conducted during the polar nights in the town of Dixon, situated on the northern latitude of $73^{\circ} 30^{\prime}$, a group of individuals with high 
helio-magnetosensitivity $(\mathrm{n}=48)$ was determined. This group underwent additional testing with the use of helio-magnetoprotective drinking water, when physiological parameters were measured three times: under the background conditions (1), 10 minutes after receiving $50 \mathrm{ml}$ of control drinking water, and on the 10th minute after receiving the same amount of helio-magnetoprotective water. Helio-magnetoprotective water was obtained after the exposure of standard drinking water in the TRODR-1 device, shielded from the geomagnetic field.

Helio-magnetoprotective means on water and holographic basis. There is a method of creating a hologram, including separation of the laser beam into the reference and object branches so that the geometric length difference between the branches did not be more than length of the coherent laser source. The disadvantage of this method is that it is not allowed to enter into the hologram nonvizualized physiologically meaningful information. ISRICA together with "Holoart" created and patented (patent RF № 2239860 from 15.11.2004) a new type of hologram recording that uses water-mineral carrier of nonvizualised physiologically meaningful information about the quantum states of different drugs. The object of the holographic recording can be in a state of active or passive transfer of information, or used as the lens of the light beam. The present study used the holograms, which are the quantum analogues of the complex of antioxidant agents.

\section{Results}

It has been shown that in conditions of increasing polar electrojets, there is a significant reduction in electrical conductivity and, consequently, an increase in electrical resistance $(p<0.01)$ in the cutaneous projections of acupuncture points (AP), which are associated with the regulation of the cardiovascular systems of the volunteers - in the points C7 and MC7 (Table I).

Table 1. The influence of polar electrojets (according to riometers) on electroparameters in the acupuncture points $(I, R, M+m)$ in healthy men $(n$ $=93)^{*}$ in the Far North

\begin{tabular}{|c|c|c|c|c|c|c|}
\hline \multirow{3}{*}{$\begin{array}{l}\mathbf{N} \\
\mathbf{n} \\
/ \\
\mathbf{n}\end{array}$} & \multirow{3}{*}{$\begin{array}{c}\text { Variatio } \\
\text { ns of } \\
\text { riometri } \\
\text { c data } \\
\text { (mm) }\end{array}$} & \multirow{3}{*}{$\begin{array}{l} \pm \mathbf{I} \mathbf{A P} \\
(\mathbf{m c A})\end{array}$} & \multicolumn{4}{|c|}{ R AP (mOm) } \\
\hline & & & C7 & & MC7 & \\
\hline & & & left & right & left & right \\
\hline 1. & $0(n=35)$ & $1.3 \pm 0,2$ & $3.5 \pm 0.7$ & $3.5 \pm 0.6$ & $2.9 \pm 0.5$ & $2.6 \pm 0.3$ \\
\hline 2. & $\begin{array}{c}0-30 \\
(n=58)\end{array}$ & $0.7 \pm 0.2$ & $1.4 \pm 0.1$ & $1.5 \pm 0.1$ & $1.4 \pm 0.1$ & $1.5 \pm 0.3$ \\
\hline $\mathrm{P}$ & & $<0.05$ & $<0.01$ & $<0.01$ & $<0.01$ & $<0.01$ \\
\hline
\end{tabular}

Notes: * - number of healthy people tested at PEP-1 device

The electrical parameters of the heart $(\mathrm{R}, \mathrm{PQ}, \mathrm{QT})$ were also dependent on the state of the magneto-ionosphere during electrocardiographic study. A significant decrease ( $p$ $<0.05$ ) of the amplitude parameters of the electrocardiogram
(R), which characterizes the energy of heart rate, was registered during strong magnetospheric disturbances (Ak 51-80). Ak is an index that characterizes in relative units the degree of changes in the components of the Earth's magnetic field and its full vector.

At magneto-ionospheric perturbations of average values (Ak 31-40) there is a significant increase $(p<0.01)$ of atrioventricular conduction, that is, increase in the interval PQ. With further increase of the amplitude of electromagnetic oscillations as a result of the inclusion of adaptation mechanisms, the electrical conductivity in the myocardium is restored (Table II).

Table 2. The electrocardiography $(M+m)$ in the volunteers $(n=120)^{*}$ depending on the state of the polar magnetosphere (according to Ak-index)

\begin{tabular}{cccccc}
\hline $\begin{array}{c}\text { Electro } \\
\text { cardio- } \\
\text { graphic } \\
\text { parame } \\
\text { ters }\end{array}$ & $\mathbf{1}(\mathbf{n = 3 4 )}$ & $\mathbf{2}(\mathbf{n = 3 1})$ & $\mathbf{3}(\mathbf{n = 1 7})$ & $\begin{array}{c}\mathbf{4} \\
(\mathbf{n}=\mathbf{3 8})\end{array}$ & $\mathbf{P}$ \\
\hline $\mathrm{0}-\mathbf{3 0}$ & $\mathbf{3 1 - 4 0}$ & $\mathbf{4 1 - 5 0}$ & $\mathbf{5 1 - 8 0}$ & \\
$\mathrm{R}(\mathrm{mm})$ & $11.55 \pm 0$. & $10.65 \pm 0$. & $10.10 \pm 0$. & $9.50 \pm 0$. & $1-4<0.05$ \\
PQ & 60 & 74 & 74 & 70 & $1-2$, \\
$(\mathrm{msec})$ & 004 & 008 & 004 & .004 & $2-4<0.05$ \\
QT & $0.376 \pm 0$. & $0.441 \pm 0$. & $0.370 \pm 0$. & $0.364 \pm 0$ & $1-2$, \\
$(\mathrm{msec})$ & 006 & 023 & 010 & .005 & $2-3.4<0.0$ \\
\hline
\end{tabular}

Notes: * - number of healthy people tested in 1999

The magnetosensitivity of the volunteers, estimated through additional magnetic testing, also appears to be dependent on the level of cosmic noise recorded by the riometer. When they increase, the value of fluctuations of blood pressure increases respectively (Fig. 1)

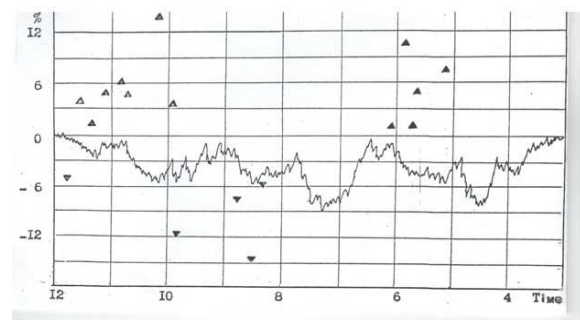

Figure 1. The intensity of fluctuations of systolic blood pressure (in \% of initial value) in the volunteers in the Far North at magnetic testing with the induction of signal $20 \mathrm{mTl}$ during periods with different levels of cosmic noise (according to riometers)

The individual features of magnetotropic reactions, defined by the vector and the degree of change in blood pressure in the same volunteers, were dependent on the concrete ionospheric situation at the time of the magnetic testing. In the perturbed state of the ionosphere, according to oblique ionographic sounding, the degree of change in blood pressure increased.

Additional testing in the selected group of helio-magnetodependent volunteers, carried out with the use of $50.0 \mathrm{~mL}$ of helio-magnetoprotective drinking water, 
showed a leveling of the functional dependence of the cardiovascular system on magneto-ionospheric perturbations: the heart chronotropic regime improved, systolic blood pressure stabilized, duration of electrical systole optimized, electrical conductivity in the cutaneous projections of acupuncture points involved in the maintenance of cardiovascular homeostasis increased (Table III). Thus the helio-magnetoprotective effect of this new non-medicinal preventive means was realized.

Table 3. Electrophysiological parameters $(M+m)$ in the subjects $(n=48)^{*}$ investigated in the Far North during disturbances of the polar ionosphere before testing(1), 10 minutes after receipt of control water (2), and 10 minutes after receipt of helio-magnetoprotective drinking water (3).

\begin{tabular}{|c|c|c|c|c|c|}
\hline \multirow[b]{2}{*}{$\begin{array}{c}\mathbf{N} \\
\mathbf{n} / \mathbf{n}\end{array}$} & \multirow[b]{2}{*}{ Parameters } & \multicolumn{3}{|c|}{ Periods of water testing } & \multirow[b]{2}{*}{$\mathbf{P}$} \\
\hline & & $\begin{array}{c}1 \\
\text { before } \\
\text { testing }\end{array}$ & $\begin{array}{c}2 \\
\text { control }\end{array}$ & $\begin{array}{c}3 \\
\text { experim } \\
\text { ent }\end{array}$ & \\
\hline 1. & $\begin{array}{l}\text { Systolic } \\
\text { blood } \\
\text { pressure }\end{array}$ & $127.3 \pm 2.4$ & $126.8 \pm 2.5$ & $\begin{array}{c}117.4 \pm 2 \\
3\end{array}$ & $\begin{array}{c}1.2-3<0 \\
.05\end{array}$ \\
\hline 2. & $\begin{array}{l}\text { Frequency } \\
\text { of systole }\end{array}$ & $67.6 \pm 2.1$ & $68.0 \pm 3.4$ & $61.1 \pm 2.5$ & $\begin{array}{c}1-3<0.0 \\
5\end{array}$ \\
\hline 3. & QT (msec) & $\begin{array}{c}0.442 \pm 0.02 \\
2\end{array}$ & $\begin{array}{c}0.445 \pm 0.0 \\
22\end{array}$ & $\begin{array}{c}0.374 \pm 0 \\
005\end{array}$ & $\begin{array}{c}1.2-3<0 \\
.01\end{array}$ \\
\hline 4. & $\begin{array}{c}\text { Electrocond } \\
\text { uctivity AP } \\
(\mathrm{I}, \mathrm{mcA})\end{array}$ & $0.7 \pm 0.2$ & $0.7 \pm 0.3$ & $1.3 \pm 0.2$ & $\begin{array}{c}1.2-3<0 \\
.05\end{array}$ \\
\hline
\end{tabular}

Notes: * - number of healthy people tested in 2012 with testing by helio-magnetoprotective water

\section{Discussion}

The following conclusions are proposed for discussion:

1. Homeostasis of the human cardiovascular system, supported with participation of non-specific electromagnetic receptors (acupuncture points), appeared to be dependent on the state of magneto-ionosphere, the intensity of electrojet, and the level of cosmic noise in the Far North.

2. Inclusion of non-medicinal protective water remedies in the drinking regime can reduce the helio-magnetosensitivity of an organism and the functional dependence of the human cardiovascular system on fluctuations of helio-geophysical factors, and can stabilize its homeostasis.

Scientists from Novosibirsk were the first to propose discussion of the hypothesis about the dependence of the health of helio-magnetosensitive persons on the activity of the magneto-ionosphere and the intensity of polar electrojets. The mechanism of prenatal helio-geophysical imprinting and its role in support of health was described [9]. It was shown that high levels of solar activity in different months of human intrauterine development were a factor in restricting lifespan and increasing the risk of different diseases $[9,10]$.

At numerous congresses on circumpolar medicine $[9,11$, 12], the Novosibirsk scientists have underlined the great role of helio-geophysical factors in the development of human cardiovascular diseases in the Far North $[11,12]$. The paper presented at ICCH-15 shows the dependence of human electromagnetic homeostasis of the cardiovascular system in the circumpolar zones on the magneto-ionosphere activity as well.

Development and testing of non-medicinal helio-magnetoprotective drink remedies seemed to be of great importance for inhabitants of circumpolar regions with high organism helio-magnetosensitivity $(60-70 \%$ of the population in Dixon). In the nearest future these remedies can be used as helio-magnetoprotectors.

While healthy volunteers were using water helio-magnetoprotectors, positive transformation of functional dependence of the cardiovascular system on the intensity of polar electrojets and cosmic noise was observed, resulting in the realization of a helio-magnetoprotective effect.

It was noted $[13,14]$ that in the conditions of repeated short-term holographic impacts many hemodynamic parameters of the volunteers $(n=16)$ improved: systolic and diastolic blood pressure decreased, pulse wave velocity decreased, endothelial function improved.

At holographic impact, the inverse correlation dependence of endothelial function on such parameters as solar activity (the number and area of sunspots) and proton component of heliogeophysical environment was revealed, i.e. at high concentrations of protons endothelial function decreased (Table 4).

Table 4. Dynamics of significant $(p<0,05)$ correlations of endothelial function in volunteers $(n=16)$ with heliogeophysical factors at holographic testing

\begin{tabular}{cccccc}
\hline & SSN & SSA & Ap & Am & Pr5 \\
\hline Background & 0,54 & 0,53 & - & - & 0,48 \\
Control & - & - & - & - & - \\
Hologram & $-0,45$ & $-0,48$ & - & - & $-0,47$ \\
\hline
\end{tabular}

Notes: SSN - sunspots number, SSA - sunspots area, Ap, Am -

geomagnetic indices, Pr5 - intensity of proton`s streams of high energy

Attention is drawn to the same type of changes in correlations of hemodynamic indices with the proton component of the different energies in the process of transition measurement from the background to the control and from the background to the test.

Thus, according to data obtained Ph.D. V. Polyakov $[13,14]$, course short-term use of holograms with analog helioprotective information (holographic glasses in 5 minutes, the source of "cold light" at a distance of $1.0 \mathrm{~m}$ ) leads to a change in the above human parameters and their interfaces with heliogeophysical factors in the direction indicating helioprotective-effect.

The data are presented, demonstrating the expressed heliomagnetoprotective effect of protracted taking of drinking water treated with the use of light -holographic technologies, which is to improve the health and optimize 
the connection of endothelial function with heliogeophysical environment.

Holographic treatment of drinking water in hypopogeomagnetic installation by way patented in Russia (Russian patent 2342149 on 27.12.2008) led to the creation of a new protective product-heliomagnetoprotective drinking water. According to $\mathrm{PhD}$ T. Kuznetsova $[13,14]$ its trial for 2 weeks on a group of volunteers $(n=8)$ under the control of the dynamics of lipid profile, which is of great importance to hemorheology, was to prove or disprove the expected heliomagnetoprotective effect in relation to the dependence of the content in the blood of lipoproteins of different density on biotropic heliogeophysical factors.

It was carried out an assessment of the dynamics and dependencies of the distribution of the inhomogeneous electron density of lipoprotein macromolecules (nanoparticles of about $10 \div 103 \mathrm{~A}$ ), of their geometric and the weight invariants in venous and capillary blood of the subjects $(\mathrm{n}=8)$ on the intensity of X-ray and radio emission from the sun, the flow of electrons with energies greater than 0.6 and more than $2.0 \mathrm{meV}$, neutrons, protons with energies greater than 1.0, more than 10,0 and more than $100.0 \mathrm{meV}$, as well as the ion plasma temperature, as measured by satellites of Goes, at the holographic testing $(\mathrm{p}<0,05)$ $[13,14]$.

A new non-medicinal way was designated to protect healthy people as well as patients with arterial hypertension, and to prevent hypertensive crisis with high risk to health and with life-threatening complications (stroke, heart attack, etc.) in periods of solar-geomagnetic disturbance. The way to produce water with heliomagnetoprotective properties with the use of information holograms in the device "TRODR" is a new developed innovational technology. The wide use of helio-magnetoprotective water opens new possibilities for the creation of global geoecological living - providing systems in the particular cosmophysical conditions that are determined by the jerks and natural geomagnetic excursions - as well as new horizons of preventive medicine of XXI century.

In 1999, work was done on themes of research approved by the Ethics Committee of SB RAMS; in 2012, on a theme approved by the Academic Council of ISRICA with the use of state and corporate funds.

The author would like to thank collaborators from the Laboratory of Helio-Climatopathology of SCCEM SB RAMS, who took an active part in the expedition work in 1999, as well as residents of the village of Dixon, who participated in the research as volunteers in 1999 and 2012.

\section{References}

[1] Mandea, M., E. Bellander, J.-L. Le Maul, “A Geomagnetic
Jerk for the End of the 20th Century," EPSI. 2000; 183: 369-373.

[2] Kuznetsov, W., "A Model of Virtual Geomagnetic Pole Motion During Reversals," Phys. Earth. Plan. Int. 1999; 115: 173-179.

[3] Newitt, LR., M. Mandea, L. A. McKee, J. J. Orgeval, “Recent Acceleration of the North Magnetic Pole Linked to the Magnetic Jerk," EOS. Transaction AGU. 2002; 83: 385-389.

[4] Breus, T.K., F.L. Komarov, S.I. Rapoport, "The Medical Effects of Geomagnetic Storms," Clinical Medicine. 2005; 3: 4-12.

[5] Stoupel, E., J. Petrauskiene, R. Kalediene, et.al., "Distribution of Death from Ischemic Heart Disease and Stroke. Environmental and Aging Influences in Men and Women," J. Basic Clin. Physiol. Pharmacol. 1996; 7: 303-319.

[6] Cornelissen, G., F. Halberg, T. K. Breus, et al., "Non-Photic Solar Associations of Heart Rate Variability and Myocardial Infarction," J. Atmosph. and Solar-Terrestrial Physics. 2002; 64: 707-728.

[7] Kaznacheev, V. P., A. V. Trofimov, "Reflections on Life and Intelligence on Planet Earth," Problems of Cosmo-Planetary Anthropoecology. Wasserburg: Academy for Future Science in Europe; 2008.

[8] Trofimov, A. V., E. V. Sevostyanova, "Dynamics of blood Values in Experimental Geomagnetic Deprivation (In Vitro) Reflects Biotropic Effects of Natural Physical Factors during Early Human Ontogeny,” Bull. Exp. Biol. Med. 2008; 146 (1): $100-3$.

[9] Trofimov, A. V., "Impact of Helio-Physical Factors on Man's Lifespan in the Circumpolar Regions. Perspectives on Intake of Helio-Geroprotectors," Int. J. Circumpolar Health. 2010; 7 (Suppl.): 356-360.

[10] Trofimov, A. V., "About the Possible Influence of Helio-Geophysical Factors in Prenatal Ontogenesis on Duration of Human Life. New Horizons of Geroprevention," Abs. of 6th European Congress of Biogerontology, Netherlands, 30 Nov-3 Dec 2008. p.75 (p. 127).

[11] Trofimov, A. V., "The Helio-Geophysical Aspects of Circumpolar Health,” Int. J. Circumpolar Health. 2001; 60 (4): 516-524.

[12] Polyakov, V., A. V. Trofimov, "Biorhythmological and Clinico-Functional Features of Arterial Hypertension under Geo-Ecological Conditions of the North," Alaska Med. 2007; 49 (2): 120-126.

[13] TrofimovAV, Druzhinin GI Information hologram: theoretical and practical perspectives for ecology and medicine of XXI century: Krasnoyarsk: Policor, 2011, 368 p.

[14] Trofimov A., Sevostyanova E. Heliogeophysical Aspects of Reology: New Technologies and Horizons of Preventive Medicine. // Rheology. New Concepts, Applications and Methods. Published by InTech, 2013. - P.39- 56. 